\title{
Hemolytic Anemia and Neurological Manifestations - An Uncommon Combination
}

\author{
Ravneet Kaur ${ }^{1}$ • Neerja Gupta ${ }^{1}$ \\ Received: 22 May 2019 / Accepted: 22 May 2019 / Published online: 10 June 2019 \\ (C) Dr. K C Chaudhuri Foundation 2019
}

Congenital hemolytic anemias are currently a major cause of morbidity worldwide, and include a wide range of phenotypically and genetically heterogenous disorders. The intrinsic red cell abnormalities include three major groups, the red cell membrane defects, hemoglobinopathies and erythrocytic enzymopathies. Despite having access to widely available basic hematological investigations such as peripheral smear, reticulocyte counts, iron levels and high performance liquid chromatography, a huge number still remains undiagnosed, and require advanced workup including molecular diagnosis. Erythrocytic enzymopathies are one of them and may present with normal red cell morphology; majority presenting as hereditary non-spherocytic hemolytic anemia (HNSHA). These may be non-neurological such as Glucose-6-phosphate dehydrogenase (G6PD) deficiency (OMIM 300908) and pyruvate kinase (PK) deficiency (OMIM 266200), and neurological. The latter includes Glucose-6-phosphate isomerase (GPI) deficiency (OMIM 613470), Triose phosphate isomerase (TPI) deficiency (OMIM 615512) or phosphoglycerate kinase 1 (PGK1) deficiency (OMIM 300653) [1]. Various neurological features in these disorders include intellectual disability, hypotonia, pyramidal tract signs, dyskinesias, ataxia, peripheral neuropathy, myalgias and progressive muscle weakness. The severity and extent of hematological and neurological manifestations varies in the neurological forms.

In this issue of the Journal, the study by Kedar et al. describes the clinical, hematological and molecular characteristics of 15 (42.8\%) unrelated patients of GPI deficiency, who presented with non-spherocytic hemolytic anemia [2]. Although, the diagnosis of other 20 patients has not been mentioned, such high

Neerja Gupta

neerja17aiims@gmail.com

1 Division of Genetics, Department of Pediatrics, All India Institute of Medical Sciences, Ansari Nagar, New Delhi 110029, India frequency of GPI deficiency amongst a cohort of cases with hemolytic anemia is difficult to explain. All of them had variable neurological involvement (intellectual disability, global developmental delay, muscular dystrophy, ataxia, neurological dysfunction) noted after infancy, except two patients who were less than one year of age, requiring long term follow up, for the later onset of neurological dysfunction. The study also shows that the presence of macrocytosis is an important feature of GPI deficiency. Though macrocytosis has been described previously with GPI deficiency, it is usually not used as a diagnostic clue [3]. Interestingly, the authors have described a simple restriction fragment length polymorphism based technique to detect a previously reported [4] pathogenic variant c.1040G $>A$ (p.Arg347His) in exon 12. This variant was observed in all 15 patients. The testing of this hotspot by a simple low cost test can be done in cases with high index of suspicion. The high frequency of the variant could be because of the high consanguinity in this cohort (12/15), inbreeding and the close geographical location of the patients, and thus, could represent a founder effect in this sub-population.

Presence of neurological phenotype with this variant and few other variants (p.His20Pro [5], p.Pro340Ser [6], IVS15(-2)A $>C$ [7] and del1473-IVS16(+2) [8]) suggest a possible genotype-phenotype correlation. The molecular basis of the neurological involvement with these variants has been previously characterized by Kugler et al. [5].

To summarize, evaluation of non-spherocytic hemolytic anemia in pediatric age group, warrants a thorough neurological evaluation to identify individuals with rare erythrocytic enzymopathies. Of these, GPI deficiency being relatively more common, should be considered as a probable diagnosis, particularly, when macrocytosis is also an associated feature. It should be followed by the testing for the highly prevalent variant c. $1040 \mathrm{G}>\mathrm{A}$ early in the diagnosis.

\section{Compliance with Ethical Standards}

Conflict of Interest None. 


\section{References}

1. Jacobasch G, Rapoport SM. Hemolytic anemias due to erythrocyte enzyme deficiencies. Mol Aspects Med. 1996;17:143-70.

2. Kedar PS, Dongerdiye R, Chilwirwar P, et al. Glucose phosphate isomerase deficiency: high prevalence of p.Arg347His mutation in Indian population associated with severe hereditary non-spherocytic hemolytic anemia coupled with neurological dysfunction. Indian J Pediatr. 2019. https://doi.org/10.1007/s12098-019-02928-1.

3. Eber SW, Gahr M, Lakomek M, Prindull G, Schröter W. Clinical symptoms and biochemical properties of three new glucosephosphate isomerase variants. Blut. 1986;53:21-8.

4. Walker JI, Layton DM, Bellingham AJ, Morgan MJ, Faik P. DNA sequence abnormalities in human glucose 6-phosphate isomerase deficiency. Hum Mol Genet. 1993;2:327-9.

5. Kugler W, Breme K, Lespe P, et al. Molecular basis of neurological dysfunction coupled with haemolytic anaemia in human glucose-6-phosphate isomerase (GPI) deficiency. Hum Genet. 1998;103:450-4.

6. Beutler E, West C, Britton HA, Harris J, Forman L. Glucose phosphate isomerase (GPI) deficiency mutations associated with hereditary nonspherocytic hemolytic anemia (HNSHA). Blood Cells Mol Dis. 1997;23:402-9.

7. Shalev O, Shalev RS, Forman L, Beutler E. GPI mount scopus-a variant of glucose phosphate isomerase deficiency. Ann Hematol. 1993;67:197-200.

8. Magor GW, Tallack MR, Gillinder KR, et al. KLF1-null neonates display hydrops fetalis and a deranged erythroid transcriptome. Blood. 2015;125:2405-17.

Publisher's Note Springer Nature remains neutral with regard to jurisdictional claims in published maps and institutional affiliations. 